\title{
Problem Analysis and New Design of TESLA Coupler
}

\author{
Ding Sun \\ Fermi National Accelerator Laboratory \\ P.O. Box 500, Batavia, Illinois 60510
}

June 1994 


\title{
Problem Analysis and New Design of TESLA Coupler
}

\author{
Ding Sun
}

\begin{abstract}
Problem analysis of tested TESLA coupler shows that the microwave discharge is a possible problem. Two new designs are presented.
\end{abstract}

\section{Analysis of the problem}

During previous tests of TESLA input coupler (doorknob with cylindrical ceramic window ) it was unable to continuously operate above $300-400 \mathrm{~kW}$ and a small area of the coupler (area A in Figure 1) where the metal doorknob and ceramic window are only $2 \mathrm{~mm}$ apart was coated with metal by unidentified mechanism. This area will be referred as area $\mathrm{A}$ in the following discussion. In order to understand the problem and help the following designs, it is necessary to analyze possible mechanisms for the problem.

(1) Electromagnetic field

In order to analyze the problem, electromagnetic field in the coupler was calculated using High Frequency Structure Simulator (HFSS) from HP company. The electric field component along the symmetric line of the waveguide (perpendicular to surface of both doorknob and ceramic in area A) varies from $\sim 500 \mathrm{~V} / \mathrm{m}$ to $\sim 300 \mathrm{~V} / \mathrm{m}$ at input power of $1 \mathrm{~W}$. The corresponding field is $\sim 0.5 \mathrm{MV} / \mathrm{m}$ to $\sim 0.3 \mathrm{MV} / \mathrm{m}$ at 1 $\mathrm{MW}$ and $\sim 0.27 \mathrm{MV} / \mathrm{m}$ to $\sim 0.16 \mathrm{MV} / \mathrm{m}$ at $300 \mathrm{KW}$. These electric fields are far below either the electric breakdown field level of the metal or the intrinsic breakdown level of the ceramic due to high electric field. Therefore other possible mechanisms should be considered than the vacuum breakdown at high field. These mechanisms are discussed in the following.

(2) Surface heating

Surface heating at metallization joints in area $\mathrm{A}$ at high power level may result in melting and evaporation of the joint material. There are three factors contributing to the extra heating in area A. (1) High current density. The computer simulation shows 
that the current density at area $\mathrm{A}$ is indeed as high as twice of the maximum current density in the waveguide. (2) Poor conductivity. The conductivity of the material for metallization of the ceramic is much lower (2-10 times) than that of good conductors. (3) Heat transfer. There is a thin metal ring joining ceramic cylinder to other metal parts. The rf currents flow on both sides of this ring while the heat transfer from the top of this ring to the base metal is poor due to the small area of cross section of the ring. Considering these three factors, the local heat at the top of the metal ring can be an order of magnitude higher than the maximum heating in a waveguide. However, computer simulation shows that the current density in area $\mathrm{B}$ ( see Figure 1) where a similar metal ring exists and no "coating" happened is as high as that in the area $\mathrm{A}$. This fact indicates that rf surface heating might not directly result in melting of metallization material. However extra surface heating can induce dramatic release of gas and thermionic emission of electrons which can result in microwave discharge (will be discussed later).

(3) Electron multipactor

Electron multipactor is resonant growth of electrons due to secondary emission. The condition for first order multipactor between two points is as follows. The total transit time for primary and secondary electrons to travel and hit opposite points should be one period of the rf field. When electrons hit the surface, the kinetic energies and incident angles of electrons must be in a certain range at which the secondary emission yield of the surface is greater than unity. The latter condition is due to the dependence of secondary emission coefficient and kinetic energy and angles of the secondary electrons on the kinetic energy and incident angels of the primary electrons.

To estimate the possibility of multipactor or cascade process (which will be discussed later) of electrons, a computer program was written to simulate electrons moving between two surfaces. Since secondary emissions from ceramic and metal depend on energy of incident electrons differently, the two surfaces in the simulation have different lower/upper limit of incident energies between which secondary emission coefficient is greater than unity. The rf field between the two surfaces can be chosen as being uniform or lineally varying. For a chosen spacing between two surfaces and initial kinetic energy of the emitted electrons (either primary or secondary), the program sweeps through 360 degrees of starting phase of the primary electrons to search possible multiplication of electrons.

For initial kinetic energy of $5 \mathrm{eV}$ and spacing of $2 \mathrm{~mm}$, the simulation results show that electric field for possible multipactor or cascade of electrons are from $0.165 \mathrm{MV} / \mathrm{m}$ to $0.42 \mathrm{MV} / \mathrm{m}$. As mentioned before, the average value of electric field component along center line of the waveguide at the area $A$ reaches those values when the power is between $170 \mathrm{~kW}$ and $1 \mathrm{MW}$. This means that multiplication of electrons in area $\mathrm{A}$ can start at power level of about $170 \mathrm{~kW}$. In order to avoid this possible multiplication of electrons the space between two surfaces may be increased. As an example, some 
of simulation results searching for the spacing above which there is no multipactor are shown in Figure 2 and Figure 3. Since only limited number of simulations were run, the data in Figure 2 and 3 should be read as that the whole area enclosed by small diamonds are the possible multipactor region. For example, Figure 3 shows that for electric field of $0.40 \mathrm{MV} / \mathrm{m}$, when spacing are between $1.85 \mathrm{~mm}$ and $4.5 \mathrm{~mm}$. the primary electrons emitted at some phases (between - 36 degree and +122 degree) can create multiplication of electrons. When spacing are less than $1.85 \mathrm{~mm}$ or greater than $4.5 \mathrm{~mm}$ there is no electrons can create multipactor at any initial phase. Figure 2 shows that for $\mathrm{rf}$ field of $0.25 \mathrm{MV} / \mathrm{m}$ there is no electron multipactor when spacing are less than $1 \mathrm{~mm}$ or greater than $3 \mathrm{~mm}$.

However, the electromagnetic field in the region between doorknob and ceramic window is not uniform and field lines are not perpendicular to the surface of the ceramic. The HFSS results show that the angle between electric field line and the normal line of the ceramic surface varies from several degrees to about 45 degrees in area $\mathrm{A}$. In such rf fields, the conditions for electron multipactor are not likely to be met. More likely, electrons which have appropriate kinetic energies and incident angles (for secondary emission yield of the surface to be greater than unity) may multiply for limited number of times and the electrons in the later stages of this cascade process will move away from the original region and finally get lost. Although the number of electrons generated in such a cascade process are much less than that in complete multipactor process, it can still reach a non-negligible level due to the high secondary emission yield of ceramic surface and initiate microwave discharge when the density of gas molecules reaches a certain level.

(4) Microwave Discharge

Microwave discharge can occur in a range of gas pressure from $10^{-4}$ torr to atmosphere pressure. In the following, it will be shown that local gas pressure in the region between doorknob and ceramic can be above $10^{-4}$ torr so microwave discharge may exist and cause the problem.

The source of gas in doorknob region is adsorbed gas molecules on ceramic and metal surfaces and absorbed gases in ceramic. The adsorbed gases on ceramic surface are several times more than that on metal surface. There are three major causes for the increase of gas molecules/atoms in the region. First, when electromagnetic wave pass through a ceramic material, the interaction between electromagnetic field and crystal lattice increases desorption of both adsorbed and absorbed gas molecules/atoms. Secondly, electrons which hit surface also increase the desorption of gas molecules. Third, rf heating discussed before can greatly increase the desorption of gas molecules. The desorption of absorbed gas molecules is a diffusion process in solid which is slow but can last for very long time. The desorption of the adsorbed molecules is an fast process. The gas particles leave the surface in the forms of molecules, atoms or ions. The molecules and atoms can be further ionized. 
As an estimation, assuming only one percent of a single layer of adsorbed gas molecules on the ceramic surface are desorbed, a simple calculation shows that the average particle density in the volume enclosed by the ceramic window can be increased to $\sim 10^{13} / \mathrm{cm}^{3}\left(\sim 3 \times 10^{-4}\right.$ torr. $)$ This is due to the high ratio of surface to volume in this region. The particle density in area $\mathrm{A}$ should be higher than the average one since the electric field and rf heating in this area is higher. In the region between doorknob and ceramic, the mean free paths of particles are limited by the dimension of the region. Thereof particles will hit the surface many times before being pumped out. At room temperature, particles can be removed by slow conditioning process. At low temperature, however, particles will be re-adsorbed onto the cold surface during the time between rf pulses ( the condensation coefficients for hydrogen and nitrogen are $\sim 0.6-1.0$ at temperature of $77 \mathrm{~K}$ ) and released again during $\mathrm{rf}$ pulses.

To initiate discharge, the average energy of electrons should be at least greater than the ionization energy of the molecules. The ionization energy of water, nitrogen and hydrogen are $12.6 \mathrm{eV}, 15.4 \mathrm{eV}$ and $15.6 \mathrm{eV}$ respectively. The average energy of the electrons for maximum ionization rate should be approximately as 2-4 times as the ionization energy. From simulation results, this condition can be met when the rf power level is above $\sim 20-40 \mathrm{~kW}$.

After initiation, both positive ions and electrons grow rapidly due to cascade process among particles and discharge may develop into arc if there is enough electrons supply. It should be pointed out that the multiplication of charged particles in this cascade process exponentially depends on the density of gas particles. The ion plasma frequency at density of $10^{13} / \mathrm{cm}^{3}$ is about $20 \mathrm{MHz}$. Therefore ions do not follow the $1.3 \mathrm{GHz}$ rf field (which also means that ions are not able to sputter material since they can not acquire enough kinetic energy.) But positive ions near the surface of either metal or ceramic can increase the electric field and electron emission from surfaces which in turn reinforce the discharge and may result in arc process. If sheath oscillation exists, it can further increase the electrons emission. During discharge and arc process, electromagnetic wave dissipate energy and electrons/ions are heated which may result in melting of the surface material and cause "coating" (actually it could be a diffusion process instead of evaporation.)

The local discharge and plasma may not cause significant reflection of $\mathrm{rf}$ power until it develops into larger scale at which the electrical property of the rf circuit can be significantly changed. This means that we should be careful during conditioning even the reflected power is small. To check this, an extra piece of conductive layer was put in region A to simulate local plasma. Shown in Figure 4 are results of some simulations. In Figure 4, the data of "Doorknob/ceramic coupler" is the simulation results of a coupler similar to the tested TESLA coupler (but the frequency for minimum reflection is $1.2 \mathrm{GHz}$ ), the data of "Coupler with $0.5 \mathrm{~cm}$ conductive layer" is the 
result of this coupler with an extra piece of conductive layer. This conductive layer is an 180 degree circular strip $(0.5 \mathrm{~cm}$ high, $0.2 \mathrm{~cm}$ thick $)$ on the inner surface of ceramic window (at the input side). The middle point of this strip is located at where the "region $A \rightarrow$ " in Figure 1 points to. The data of "Coupler with $1 \mathrm{~cm}$ conductive layer" is the result of this coupler with a similar conductive layer (but the height of the conductive layer is $1 \mathrm{~cm}$.) It should be pointed out that the conductance of plasma being discussed is lower than that of conductive layer, therefore there is even less reflection from a real plasma.

\section{New Design of Coupler}

In order to avoid the above discussed problem and make conditioning process relatively easier, basically there are two options for new design of couplers: (1) to replace the cylindrical ceramic window with a planar waveguide window or a disc/cone coaxial line window which is not close to the metal doorknob coupler and (2) to modify the doorknob part or use larger ceramic window to increase the space between ceramic cylindrical window and coupling part. Besides, the coupler should have broad band width.

Following these directions, two couplers were designed recently. One is a doorknob coupler without ceramic window and the other has ceramic window with a simple coupling post. The basic results are presented in the following.

(1) Doorknob coupler

Shown in Figure 5 is the doorknob coupler. The feature of this doorknob coupler is its low SWR, broad band and relatively not sensitive to the geometry variation.

Sown in Figure 6 is the SWR (standing wave ratio) of this coupler versus frequency calculated by HFSS. The minimum SWR at $1.3 \mathrm{GHz}$ is 1.05 . The band width is 120 $\mathrm{MHz}$ for SWR being below 1.2 and $200 \mathrm{MHz}$ for SWR being below 1.5.

According to literatures, performance of doorknob couplers are very sensitive to the geometry change. Thereof the variation of the $S$ parameters of the designed coupler with the change of the doorknob geometry are examined. Some of the results are shown in Figure 8 . Because it is difficult to describe a doorknob with simple parameters, the doorknob shown in Figure 5 was designed as a combination of three basic parts (see Figure 7): (1) a cylinder (named as cylinder), (2) a quarter of a circle resolved along $Z$ axis (named as circle 1), and (3) another quarter of a circle resolved along $\mathrm{Z}$ axis (named as circle 2). The $\mathrm{Z}$ axis is the center axis of coax port. With these three parts, the geometry of the designed doorknob can be changed by adjusting the radius or height of these three parts. Shown in Figure 8 are $\mathrm{S11}$ data (simulation results) of 4 couplers. The difference among the 4 couplers are as follows. Relative 
to DS26, the radius of the circle 1 was decreased by $2 \mathrm{~mm}$ while the radius of the circle 2 was increased by $2 \mathrm{~mm}$ in DS46, the height of the cylinder was increased by $2 \mathrm{~mm}$ in DS56, the radii of both circle 1 and 2 were decreased by $1.5 \mathrm{~mm}$ and height of the cylinder was increased by $1.5 \mathrm{~mm}$ in DS27. These results show that the designed doorknob coupler (which is close to DS27) is not sensitive to small geometric variation. The S11 values of all four versions are acceptable. In practice, the error due to machining can be less than 5 mils and the error due to assembling can be less than 10 mils which are 6-8 times smaller than the variation used in our examination.

During the cavity filling time there will be a full reflection. To check the maximum field during this filling time, the HFSS was performed with the coaxial section shorted at various places. The maximum field at the surface of the doorknob are less than 3 $\mathrm{MV} / \mathrm{m}$ which is far below the $\mathrm{rf}$ breakdown field at $1.3 \mathrm{GHz}$ in vacuum.

(2) Coupler with cylindrical ceramic window

Shown in Figure 9 is the new coupler with cylindrical ceramic window. The feature of this coupler is the simplicity of the geometry and increased spacing between the ceramic window and the coupling post. The spacing has been increased to $1.5 \mathrm{~cm}$ while the narrow spacing between the doorknob and ceramic cylinder in the tested TESLA coupler is about $2 \mathrm{~mm}$. These features will provide two advantages: (1) the density of the gas molecules released from ceramic surface due to the electrons induced desorption and of heating will be lower which will reduce the possibility of discharge or arc, (2) the possibility of electron cascade process due to secondary emission will be eliminated as discussed in section I..

Shown in Figure 10 is the SWR of this coupler which is 1.003 at $1.3 \mathrm{GHz}$. The band width is $\sim 60 \mathrm{MHz}$ for SWR being bellow 1.2 and $\sim 140 \mathrm{MHz}$ for SWR being below 1.2. 


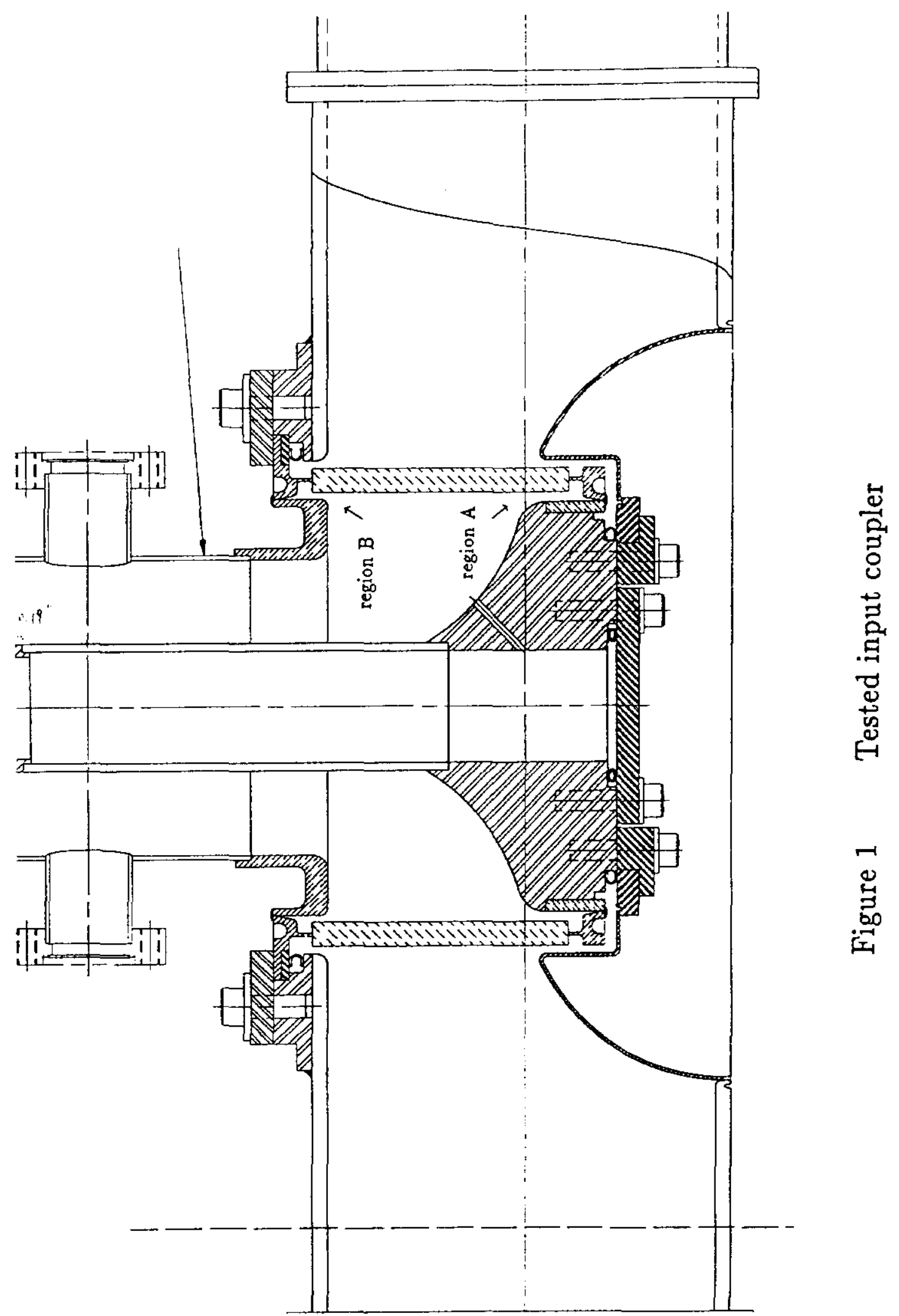




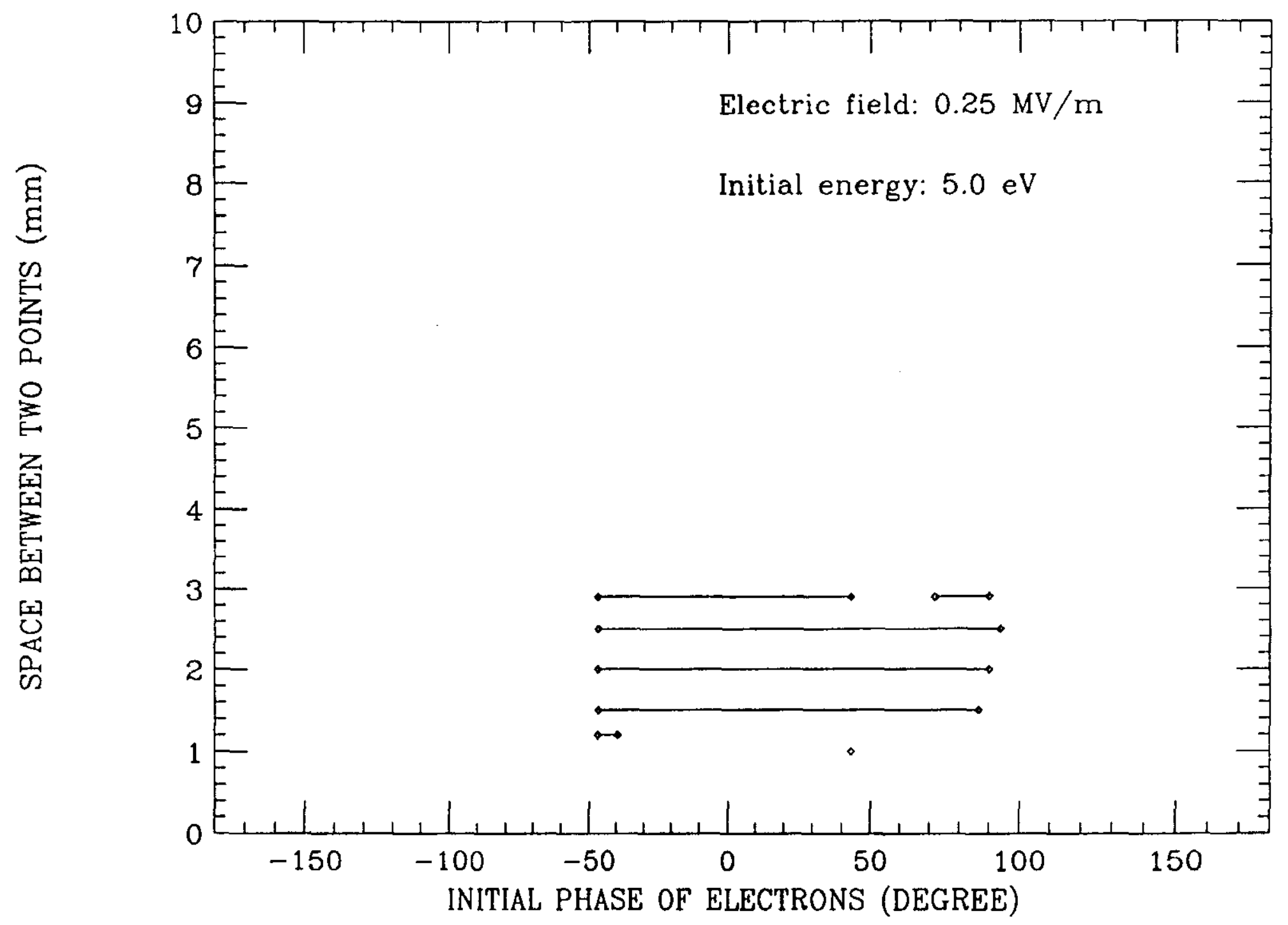

Figure 2 Multipactor region between two surfaces 


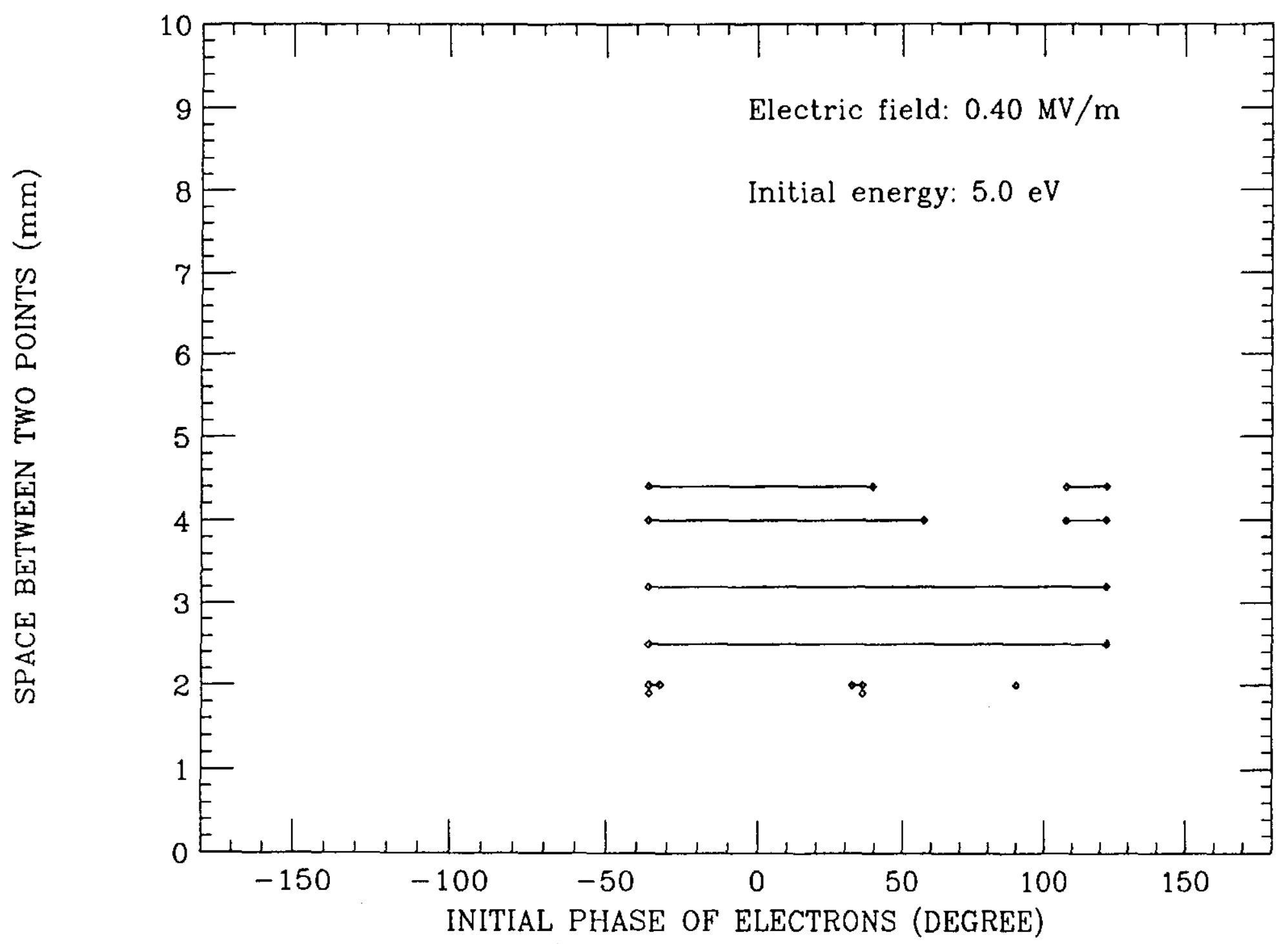

Figure $3 \quad$ Multipactor region between two surfaces 


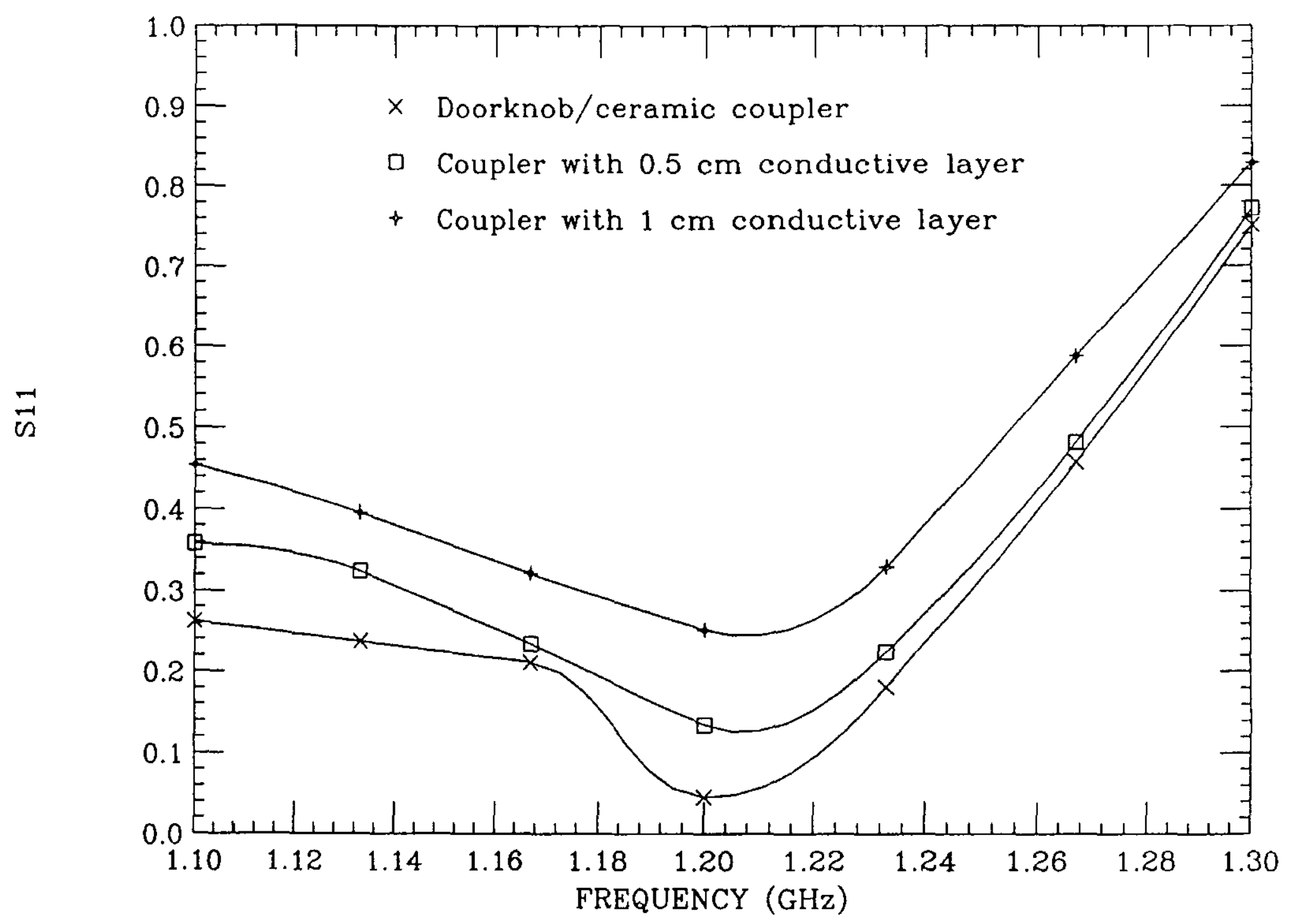

Figure 4 Reflection due to conductive layer 


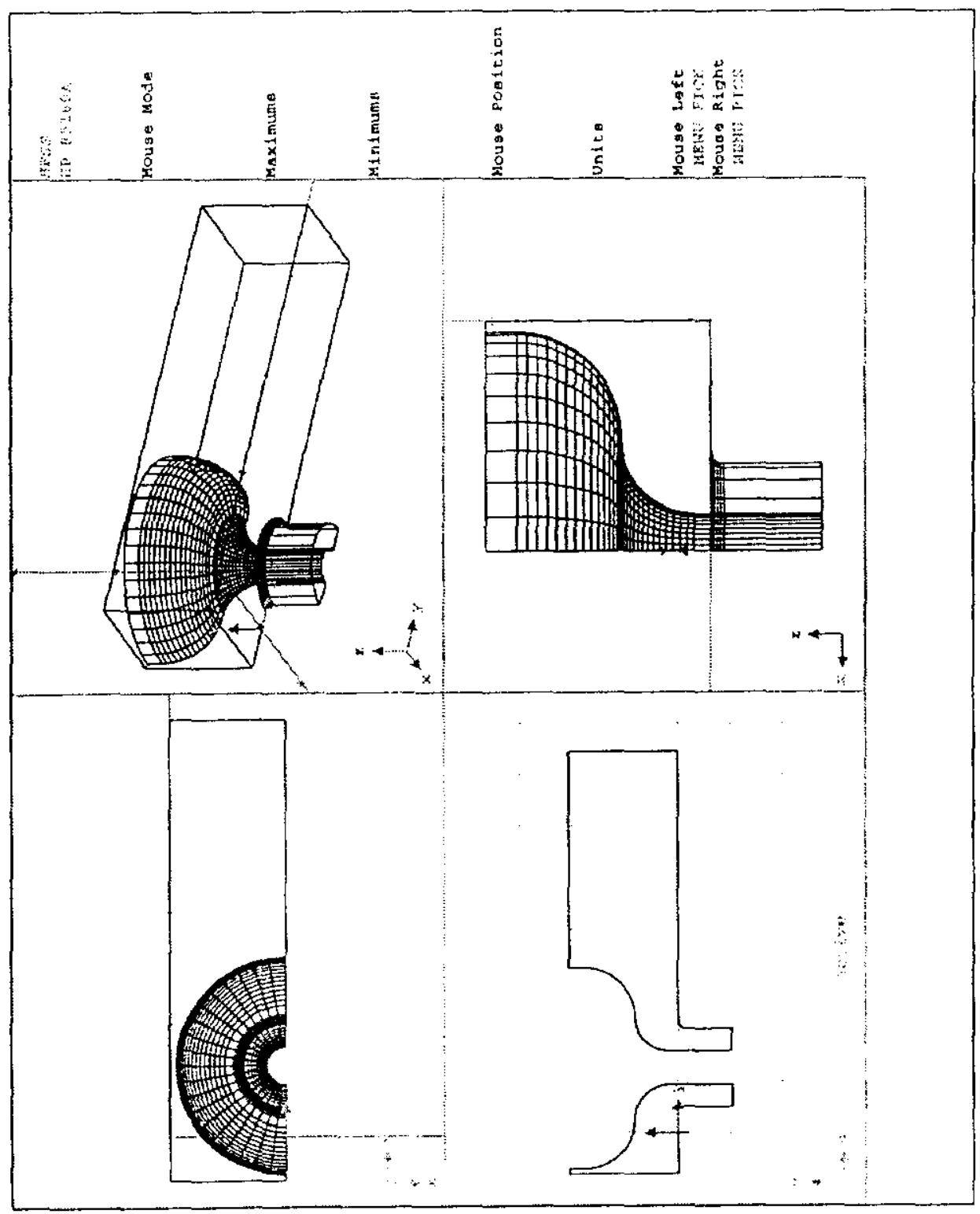

4
0
0
0
0
0
0
0
4
4
0
0
0
3
0
$z$

10 


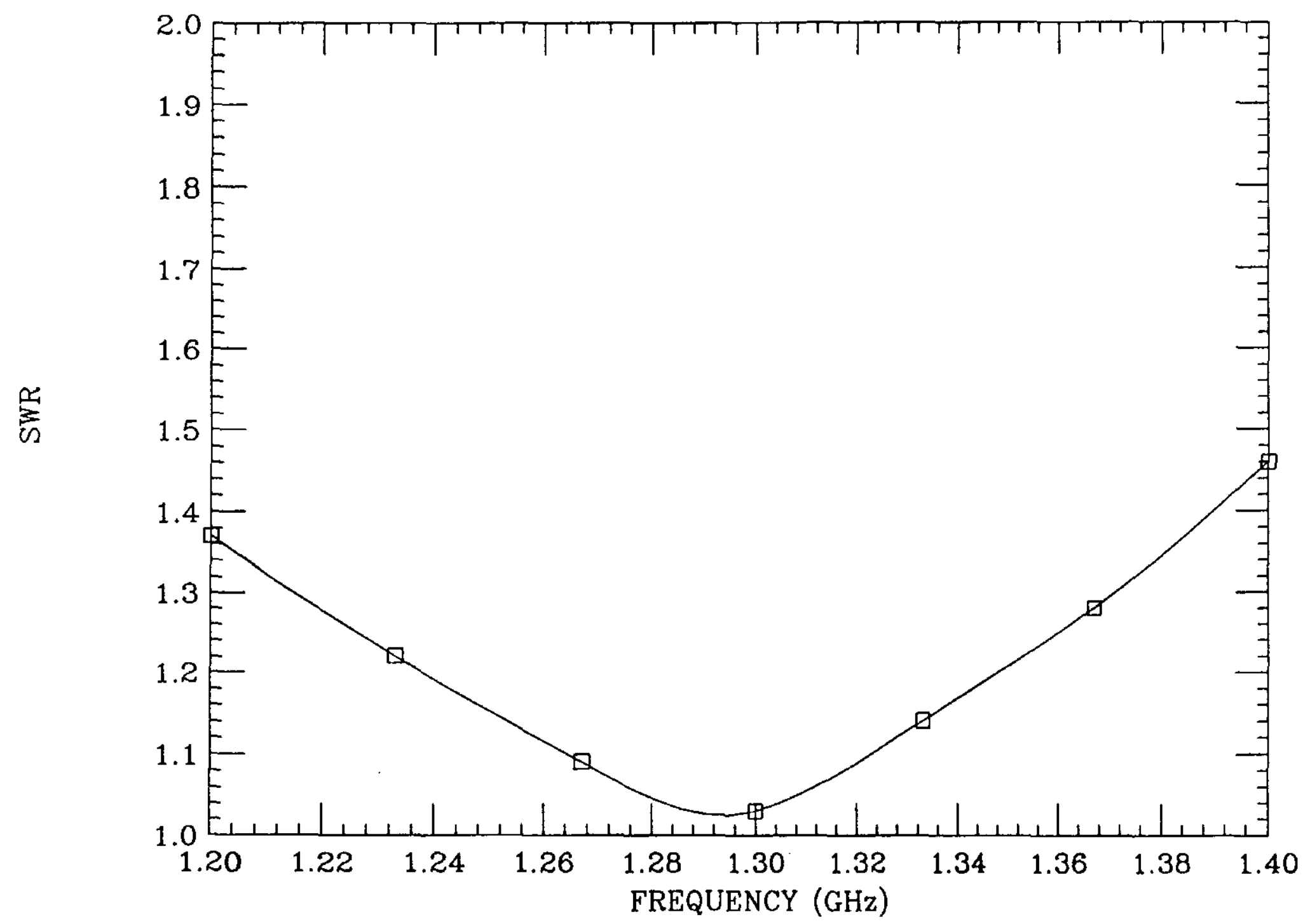

Figure $6 \quad$ SWR of new doorknob coupler 


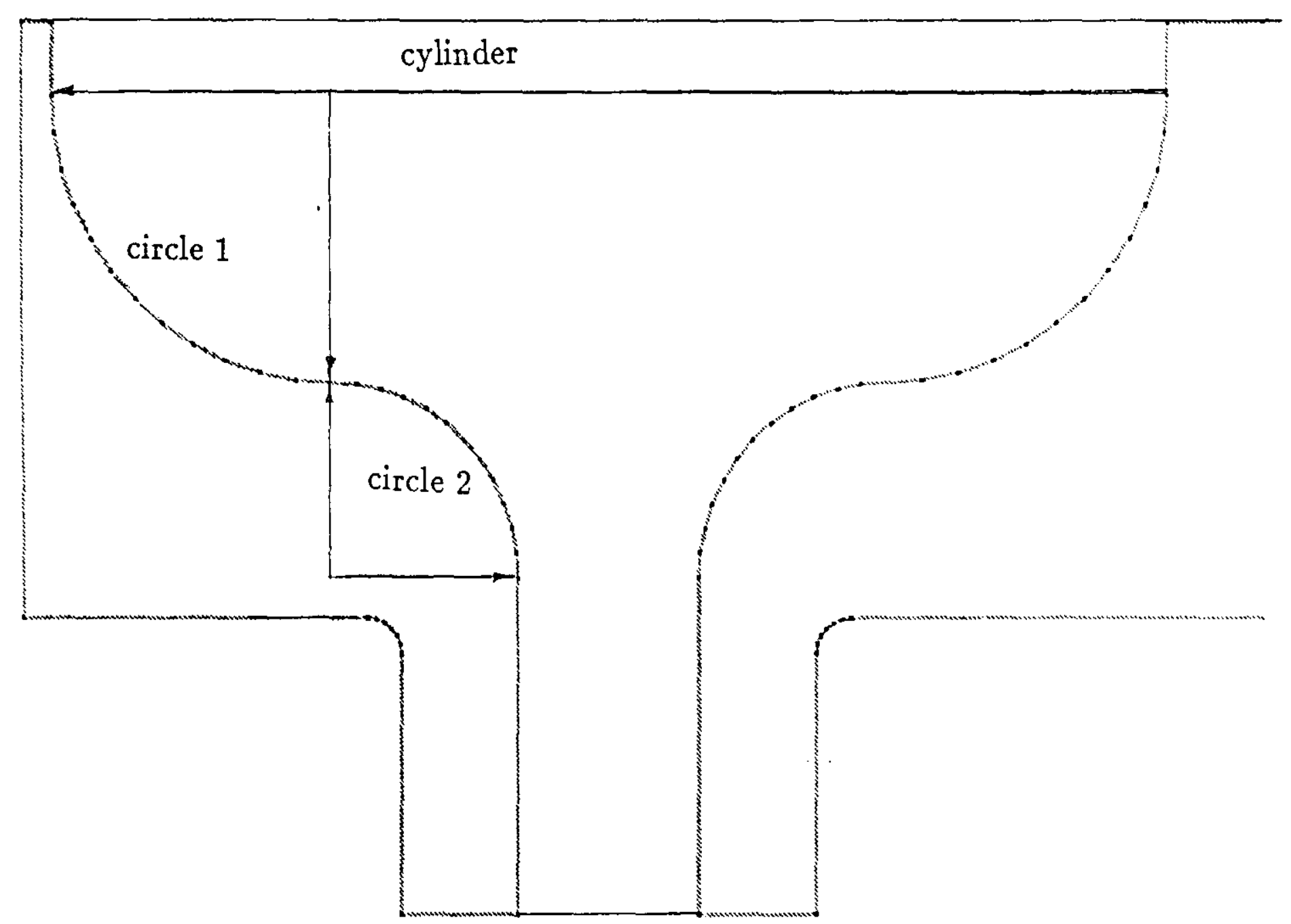

Figure $7 \quad$ Basic blocks of doorknob coupler 


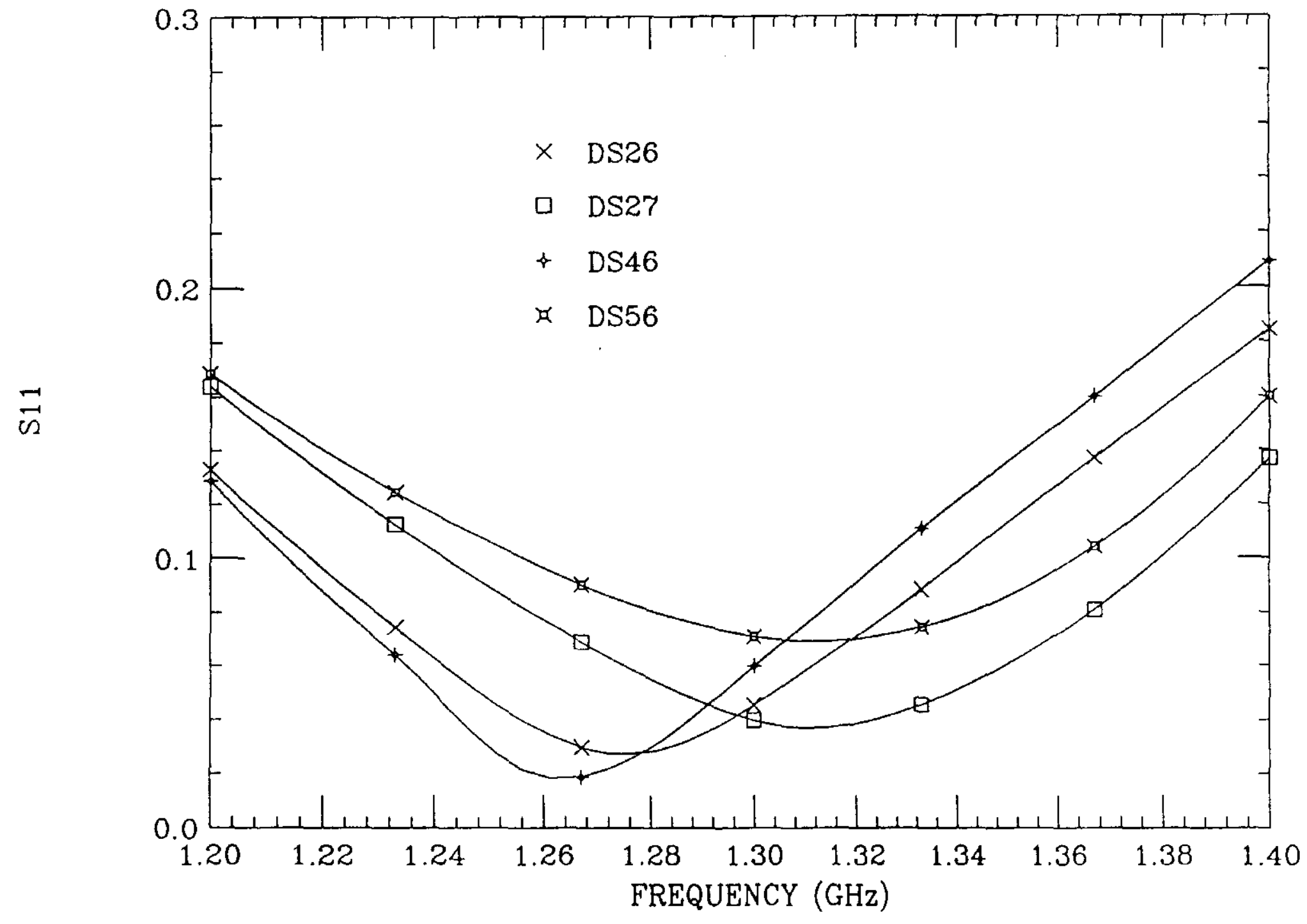

Figure $8 \quad \mathrm{~S} 11$ of four doorknob couplers 


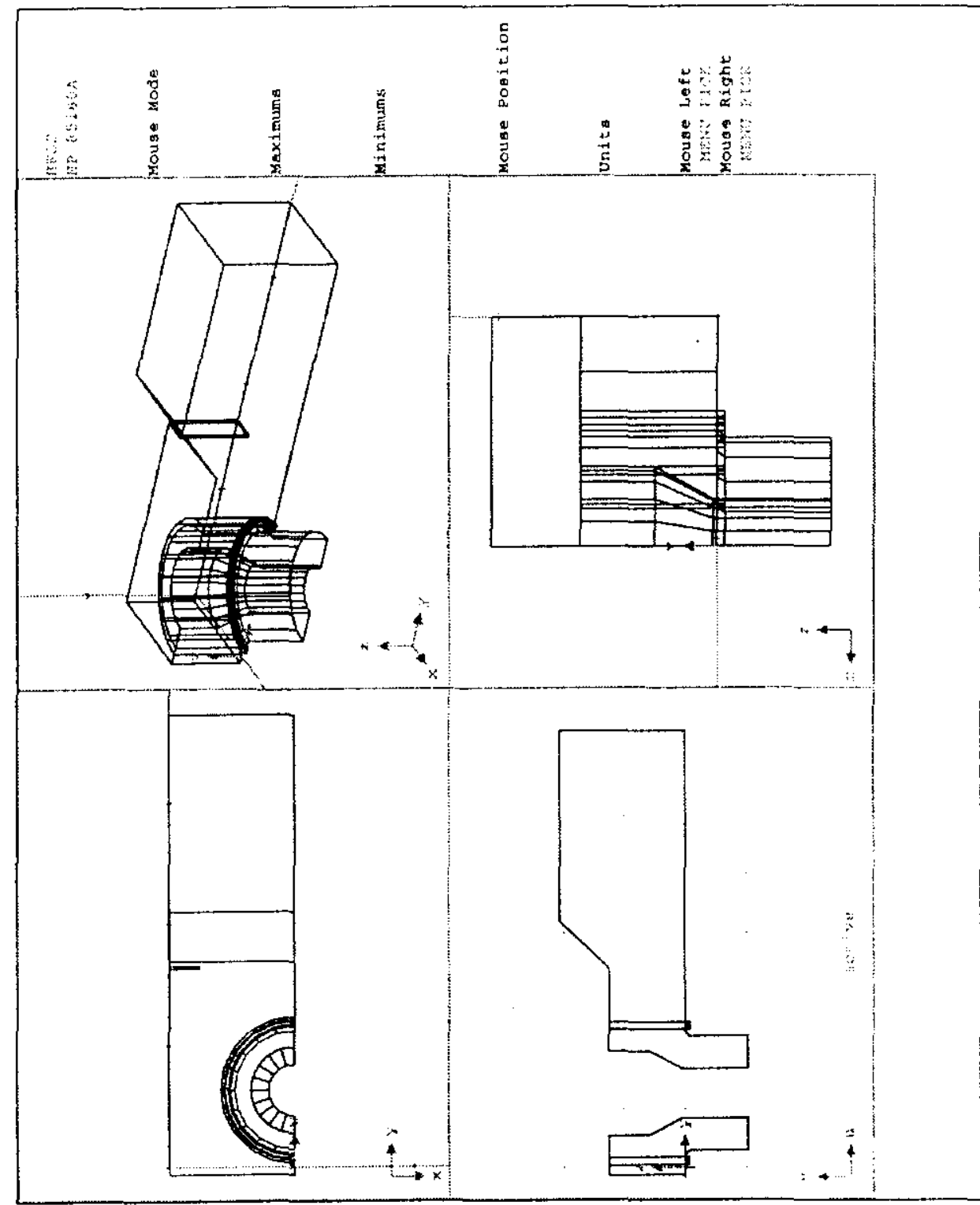



0
0
0
0
0
01 


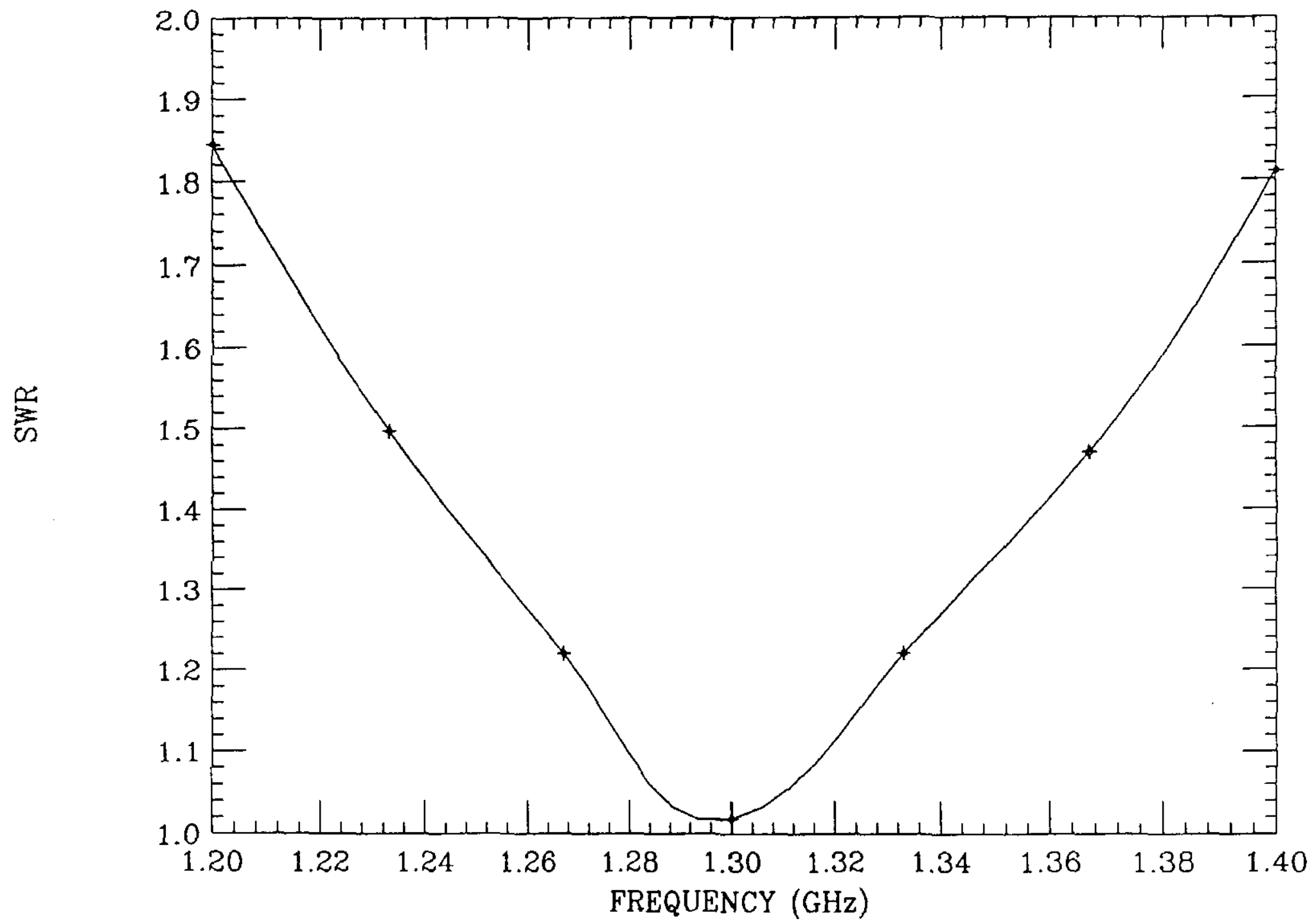

Figure $10 \quad$ SWR of new coupler with ceramic window 